\title{
Acquiring high value, retainable customers
}

Received (in revised form): 24th May, 2004

\section{Andrew Banasiewicz}

is a Vice President of Analytic Services at Boston-based Hill, Holliday Advertising. Prior to joining Hill, Holliday, Dr Banasiewicz served as a Vice President of Strategy and Analysis at Digitas LLC, Director of Analytical Consulting at Epsilon, Inc. and Advanced Analytics Manager at AC Nielsen. He also served on the faculties of Louisiana State University and the University of Wyoming. He holds a PhD in business analysis from Louisiana State University. His current work focuses on analytic planning and the design of custom decision support and measurement systems for marketing. His background includes research design and statistical analysis of primary and secondary data sources; database development and modelling; measurement and experimentation; survey design and analysis; and UPC scanner data analysis. He has authored several methodological and topical articles, including 'Keeping Your Best Customers Through Brand Loyalty', 'Selecting a Segmentation Approach' and 'How to Identify High Value Customers'.

Abstract The often cited poor productivity of new customer acquisition spending is in large part due to methodological neglect, particularly the ineffectiveness of the open offer-centric customer recruitment programmes. The author proposes an alternative, information-driven customer type-centric acquisition framework designed to increase the yield of acquisition marketing expenditures by leveraging the already available data assets. The proposed framework is built around strategic alignment and methodological parity of customer acquisition and retention efforts, bringing to light the benefits of targeted acquisition informed by retention trends. By dissecting the acquisition process itself, the analysis underscores the dangers of over-reliance on the discount promoting open offers, discussed in the context of adverse consumer selection. Lastly, addressing the need for accurate high value customer identification, the author presents an alternative operationalisation of that key metric, leading to the construction of the acquisition spending allocation matrix.

Andrew Banasiewicz Vice President of Analytic Services,

Hill, Holliday Advertising, Inc., 200 Clarendon Street, Boston, MA 02116, USA.

Tel: +1 6175853613 ; Fax: +1 6175853813 e-mail: abanasiewicz@hhcc.com

\section{INTRODUCTION}

New customer acquisition tops the marketing agenda of most organisations. After all, the ever-present attrition and the equally ubiquitous growth expectations make customer recruitment a virtual necessity. Yet, in spite of its apparent importance, acquisition as a marketing practice area remains methodologically neglected. Overreliance on convenience populations such as third party consumer lists coupled with generic purchase incentives and off-the-shelf consumer valuation/targeting schemes - continue to handicap its effectiveness. Even more importantly, the focus on a 'head count' near-term programme response - as the de facto acquisition success metric, and the resultant inattentiveness to longer-term product repurchase and customer retention, continue to impact adversely the productivity of customer recruitment efforts.

Many of the acquisition shortcomings are encapsulated in its most common tool, a discount-based open (ie one available to all consumers) offer, best exemplified by 'cash back' in the auto industry or manufacturers' coupons in 
consumer goods. Not only are these offers weighing down transactional profitability, but they can also contribute to longer-term attrition through the heightening of consumer price sensitivity. Overall, tying new customer acquisition to ongoing discounting increases the dependence of brands' sales volume on price reductions, thus negatively impacting the overall customer base profitability.

Undeterred by high costs and low yields, companies continue myopically to pour significant resources into ineffective customer recruitment efforts. Acquisition managers rarely look past the initial brand trial, their eyes glued to near-term promotional response as the success metric. For one reason or another, marketers endure in their belief that the initially discounted brand samplings spark full price repurchase on a large enough scale to warrant the often hefty acquisition investments.

Working with organisations in the consumer/packaged goods, automotive, retail, financial services and other industries, the author found limited support for that rationale. In fact, the few instances where he encountered supporting evidence involved relatively specific, well-defined consumer segments. More often than not, the net sum of programme-attributable incremental sales gains did not justify the overall programme expenditures.

Adverse customer selection, which is the tendency of the least desirable consumer types to show the highest brand interest, bears much of the blame. The name itself may sound new, but the trend of price sensitive brand switchers exhibiting the strongest propensity to respond to a discount-based brand trial offer is well known to many. Equally familiar is the frequently seen consequence of adverse selection, which is that the initially attractive near-term promotional response and brand trial rates are often followed by low full price repurchase, this is illustrative of brand switchers not sticking around without continued subsidies. In the end, considerable up-front acquisition programme expenditures routinely deliver unacceptably poor longer-term returns, leaving in their wake no more than a handful of brand repurchasers acquired at an excessively high per customer cost.

The doggedly poor performance of new customer acquisition programmes can be traced back to a couple of interrelated factors:

- the practice of approaching acquisition as a stand-alone marketing function; and

- over-reliance on open offer discounting.

Starting with the former, when treated as a self-contained effort, new customer recruitment takes on a near-term focus, as evident in the initial brand trial being the primary success metric. The longer-term brand benefits, such as the overall customer base profitability, are not fully considered. As a consequence, random customer self-selection takes the place of systematic customer base value replenishment as a means of combating the ongoing attrition. This is of pivotal importance to the overall customer base and will be covered in more detail later.

The second key cause of acquisition ineffectiveness is the proliferation of price discounting-based incentives. As briefly mentioned above, open discounts not only take away from transactional profitability but can also bring about the heightening of consumer price sensitivity. Although perhaps less immediately, that can lead to further exacerbation of the customer attrition problems initially created by adverse customer selection. In the end, acquisition programmes built 
around generic discounting have limited longer-term benefit. This is not to say that temporary price discounting should be abandoned - it should, however, be used in a more targeted fashion.

Taking a bigger picture view, any increases in acquisition productivity are contingent on the recasting of its objectives in the context of longer-term customer base growth and profitability rather than a mere near-term brand trial. Of course, it is not an easy task simply because it requires a considerable philosophical and methodological mind shift away from the 'mass-marketed' acquisition and towards information-driven, targeted and differentiated programmes. In other words, the acquisition paradigm needs to evolve from a generic discount focus to consumer type centeredness.

Naturally, a change of that magnitude can only happen in a stepwise manner, with the first step being an explicit recognition of the crucial acquisition-retention link. With that as a foundation, other, more operationally minded steps should follow, involving consumer targeting and offer differentiation considerations. The notions of transaction and customer base level profitability; customer base replenishment; and strategic alignment and methodological parity will all play a pivotal role in the development of an acquisition transitioning road map. The overall process, as well as the individual components, are outlined in the ensuing sections of this paper, starting with an in-depth overview of the retention-acquisition link.

\section{THE RETENTION-ACQUISITION LINK}

As previously suggested, the most striking trait of new customer acquisition programmes is their lack of emphasis on repurchase and transaction-level profitability. Most are designed to drive brand trials through unsustainable discounts, drawing customers to discounts, not the brand itself. Not surprisingly, many of the initially 'successful' acquisition programmes turn out to perform quite poorly when evaluated in the context of longer-term repurchase or profitability. It seems obvious that it is near-sighted to equate acquisition success with discounted product trials, as it does not account for post-trial repurchase nor does it reflect transaction level profit contribution. To be worth the investment made in it, new customer acquisition needs to generate acceptably high numbers of near-term product trials as well as acceptably high longer-term, full price repurchase rates. In short, acquisition needs to be closely integrated with retention.

The key to seamless acquisition-retention integration is the notion of customer attrition loss replenishment. This concept reflects the ubiquitous nature of customer attrition - an ongoing customer loss either to competitors or due to 'natural' causes such as customer lifecycle. In other words, some level of attrition is unavoidable. This precipitates the following questions: How effective are the current new customer acquisition efforts in replenishing customer base losses? More specifically, if disproportionately large numbers of high value customers are leaving the franchise, are their replacements of equally high quality? These questions highlight an important dimension of new customer acquisition, namely attrition replacement and the importance of attracting well chosen - not just any - customers.

Let us not forget the better known acquisition objective, which is that of driving near-term initial brand trials. Joining these two objectives together, 


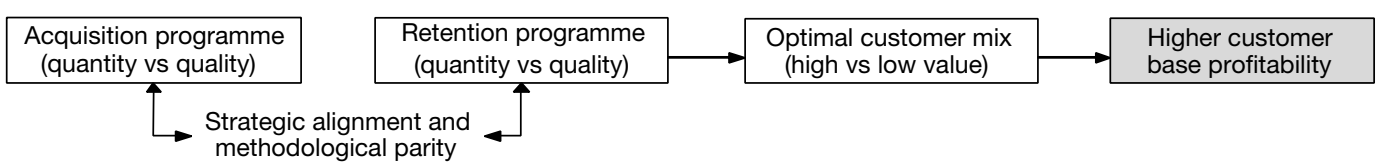

Figure 1: Customer mix optimisation

namely the customer attrition loss replenishment and initial brand trials, the value of avoiding the trap of initially strong promotional response followed by dismal repurchase rates becomes even more pronounced. In short, acquisition and retention need to be treated as two related parts of the same process, rather than as two separate processes. In analytical terms, the two should jointly contribute to the optimisation of the customer mix through strategic alignment and methodological parity, as shown graphically in Figure 1.

Figure 1 depicts a couple of acquisition 'musts'. First and foremost, programme requirements need to be stated in terms of customer base objectives. Brand trials should not be viewed as the end objective of acquisition, as the often-subsidised initial purchase is rarely profitable or, for that matter, even indicative of future full price repurchase. A stable and profitable customer base, not just a one-time brand trial, should be the ultimate goal of new customer acquisition. That goal, however, cannot be realised if acquisition continues to be treated as a stand-alone process. To be effective as a business tool, it needs to be thought of as means of attracting tomorrow's high value loyal buyers, which, in turn, necessitates the retention-acquisition integration. As shown in Figure 1, the means to that end are the notions of strategic alignment and methodological parity.

Given their importance, strategic alignment and methodological parity warrant deeper explanation. The former is a two-pronged requirement. First, it calls for an explicit delineation of the current customer base structure, attrition trends and velocity as well as the most effective retention magnets. At a more global level, it requires a retention and acquisition shared brand positioning vision, clearly communicated in the respective programmes' targeting decisions and messaging themes. In other words, strategic alignment is a manifestation of acquisition and retention programmes' shared level of customer base understanding and goal coordination.

The more operationally minded methodological parity encapsulates communality of buyer type segmentation schema and the basic analytical structure of differential treatment and investment allocation guidelines. Acquisition and retention should be two branches of the same methodological tree, sharing common segmentation and treatment methodologies. The benefits of it include maximising the potential of the customer base, ultimately manifesting itself in higher customer base profitability.

At least to some readers, acquisition-retention strategic alignment and methodological parity may sound alarmingly complex. When contrasted with 'one size fits all' programmes built around generic discounts, integrated acquisition programmes are indeed more complex to design and execute, especially since their end objective goes far beyond initial brand trials. Some of the otherwise nonexistent obstacles include data modelling requirements, the development of multiple differentiated offers, consumer segment specific 


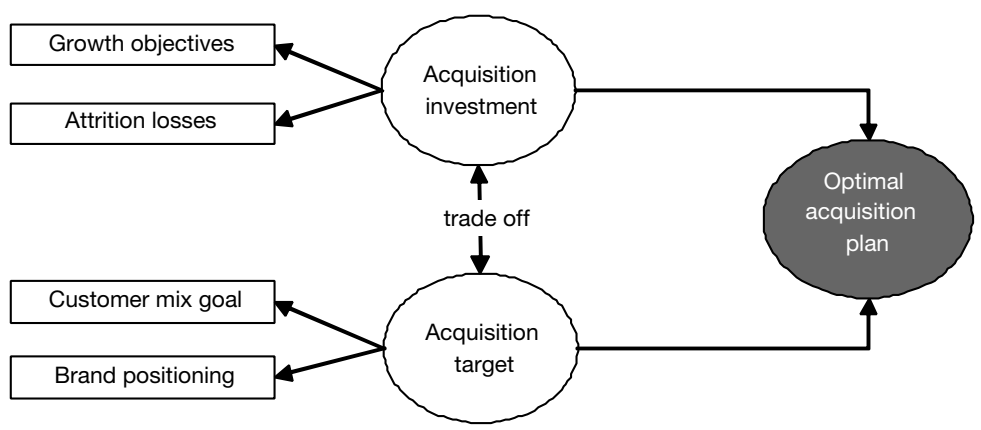

Figure 2: Components of the optimal acquisition and their interdependencies

investment reallocation, or impact measurement expectations. On the other hand, these programmes deliver superior results, ranging from higher initial response rates (two-fold and higher increases are common), to higher long-term repurchase rates (ie $2-4 \times$ increases in the automotive or financial services industries) to encouraging programme return on investment (ROI) (usually highly positive). The reason behind their success and higher level of difficulty is the information driven customer mix development.

\section{CUSTOMER MIX OPTIMISATION}

Approaching acquisition as a customer base optimisation process forces a brand manager to look beyond the number of initial product trials (ie the possibly misleading 'head count') and consider the long-term revenue stream as the ultimate goal of new customer acquisition. In doing so, customer acquisition planning evolves beyond offer-centricity and customer self-selection and into targeted high potential value buyer recruitment. Generic, open offers built around temporary price reductions are replaced with offers tailored to specific consumer types and targeted at 'pre-qualified' consumers only. Instrumental in this transition is an explicit delineation of the key acquisition drivers of targeting and investment allocation, which, in turn, requires clearly stated growth objectives, attrition loss accounting, future customer mix goals and an operational level of brand positioning understanding. Figure 2 outlines the individual components as well as their interdependencies.

Acquisition investment represents the desired quantity of the net expected customer base increase, expressed as a function of growth objectives adjusted for attrition losses. Acquisition target, on the other hand, embodies the desired quality of recruited customers, which is a function of customer mix objectives and brand positioning. The trade off between acquisition investment and acquisition target reflects the 'give and take' between the required level of investment and the stated acquisition target objectives.

The customer acquisition process outlined above goes beyond mere replenishment of quantities as it explicitly addresses quality considerations - in doing so, the ineffective discount orientation is replaced with customer type orientation.

For example, if, during a given time period, buyer loss is skewed towards high value customers, the customer type-focused acquisition takes into account both the raw replenishment needs and customer type-specific goals, thus balancing near-term 'head counts' 
with longer-term retention and customer base profitability. Its impact, particularly when contrasted with an open offer-based acquisition, can be substantial, as illustrated by the following hypothetical case.

\section{Offer-centric acquisition scenario}

An open offer programme's likelihood of acquiring a high value customer is approximately equal to the overall proportion of those buyers in the product category. Given that, on average, high value customers account for 10 per cent or less of all buyers, an open offer programme's likelihood of attracting a high value customer is 10 per cent or less. That means that if a given brand is losing its high value customers at a rate higher than 10 per cent, open offer-based new customer acquisition will not be able to keep up with high value customer attrition, leading to a gradual erosion of the overall customer base profitability. All that while in near term it may generate sufficient offer response 'head counts'.

\section{Customer type-centric acquisition}

The above scenario is exactly what the customer type-centric acquisition is trying to circumvent. It does so by bringing to bear current attrition trends and other related inputs to set specific customer type acquisition targets (see Figure 2), to be followed by differential treatment (discussed later in more detail). For instance, high value customer attrition of 10 per cent translates into a clear replenishment goal, which is supported by consumer profiles and targeted/differentiated messaging and offer strategies. Adverse consumer selection is minimised and sustained or growing customer base profitability is a matter of working known means towards clearly stated objectives. Customer type-centric acquisition replaces uncertainty and guesswork with factual information supporting well-defined marketing actions, all without increasing the overall cost.

The remainder of this paper outlines the process for migrating from the open offer acquisition approach to the customer type focused one. For comparative purposes, a brief summary of the former is presented first, followed by a more detailed discussion of the latter.

\section{A SUMMARY OF THE PITFALLS OF OPEN OFFER ACQUISITION}

A widely used mass acquisition approach, the open product trial offers, relies on temporary price discounts to pull consumers to the brand. Trying to appeal to a generic many rather than a select few consumers, these programmes are relatively easy to execute but yield poor ROI. They rarely include explicit retention considerations which inhibits their ability to stem longer term customer base profitability erosion. Consequently, customer acquisition built around open offer programmes will suffer from the following:

1 Inflated 'per acquired customer' costs. Large prospect pools, multiple channels, over-reliance on price incentives and low response rates all add up to a considerable financial outlay made on every newly acquired customer.

2 Poor repurchase and retention rates. As outlined previously, self-selected brand switchers comprising the majority of the open offer-attracted new customer pool are very likely to defect in the absence of considerable ongoing price discounts.

3 Poor transactional value. High per customer acquisition costs coupled 


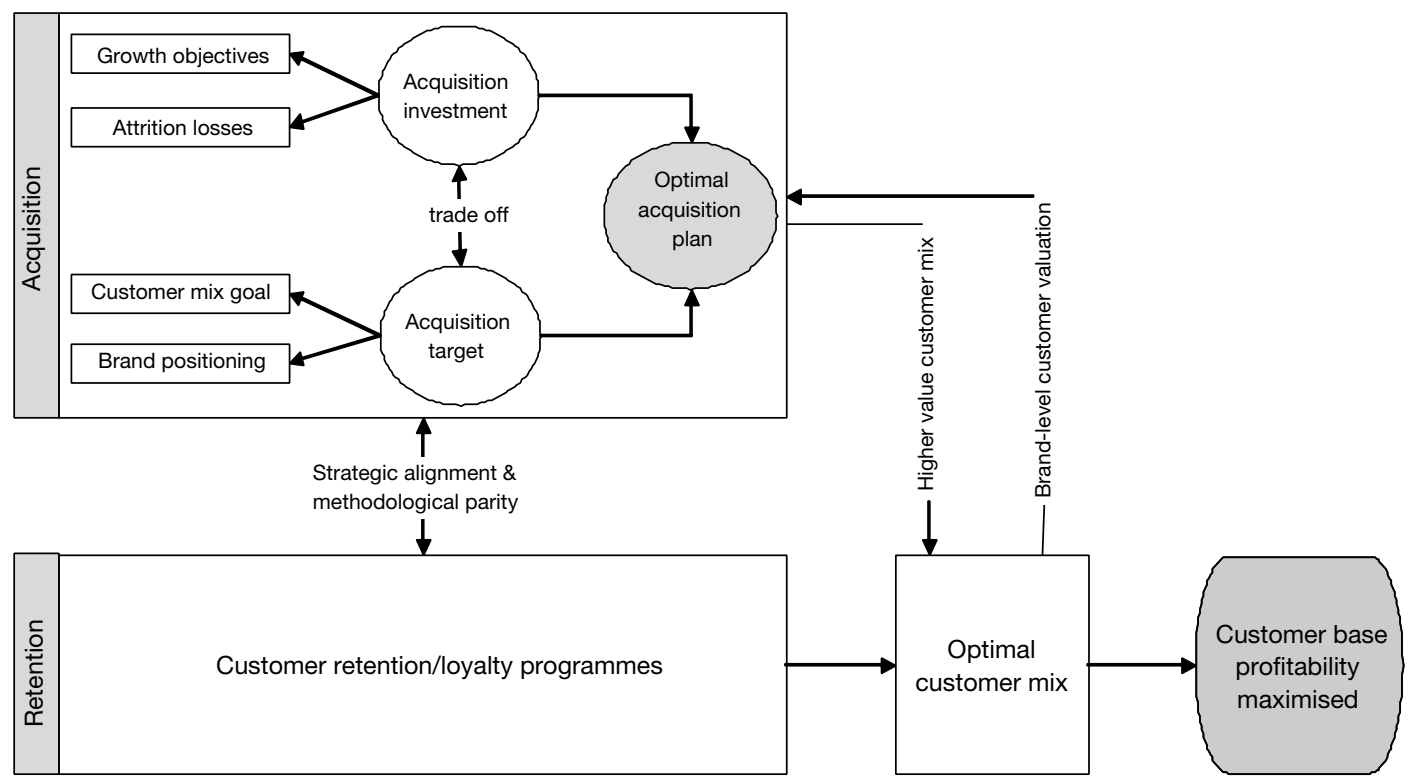

Figure 3: Customer type-centric acquisition framework

with high attrition propensity make this mode of acquisition relatively unprofitable in both the near and long terms.

4 Heightened likelihood of long-term customer base profitability erosion. As exemplified above, open offer-based acquisition is a game of odds, but one where the odds are stacked against it to begin with, as the high value customer recruitment rates associated with those programmes are usually lower than most brands' high value customer attrition rates.

\section{CUSTOMER TYPE-CENTRIC ACQUISITION}

Price discount habituation, low transaction level profitability, poor retention and potential customer base profitability decay are all sufficient reasons to consider an alternative to open offer-based customer acquisition. The previously outlined customer type-centric methodology is one such alternative. Fundamentally different from the open offer-based acquisition, this approach takes a broader view of the customer recruitment process by expressly relating the near term incentive-based product trial, longer term post-trial repurchase and the overall customer base profitability. Built around the notions of strategic alignment and methodological parity between customer acquisition and retention (see Figure 1), the customer type-centric acquisition approach expands upon the key acquisition considerations depicted in Figure 2, by operationalising consumer value in a way that supports explicit differentiation between category-wide spending levels and brand-specific conversion propensity. The alternative acquisition methodology is outlined in Figure 3.

The acquisition methodology shown above is rooted in several key considerations. First of all, it promotes the belief that to be efficient and economically sound, acquisition should be focused on targeting the right - not all - high value consumers. A common error is to define an acquisition prospect's value in terms of category-wide purchase levels, thus implicitly assuming that all 


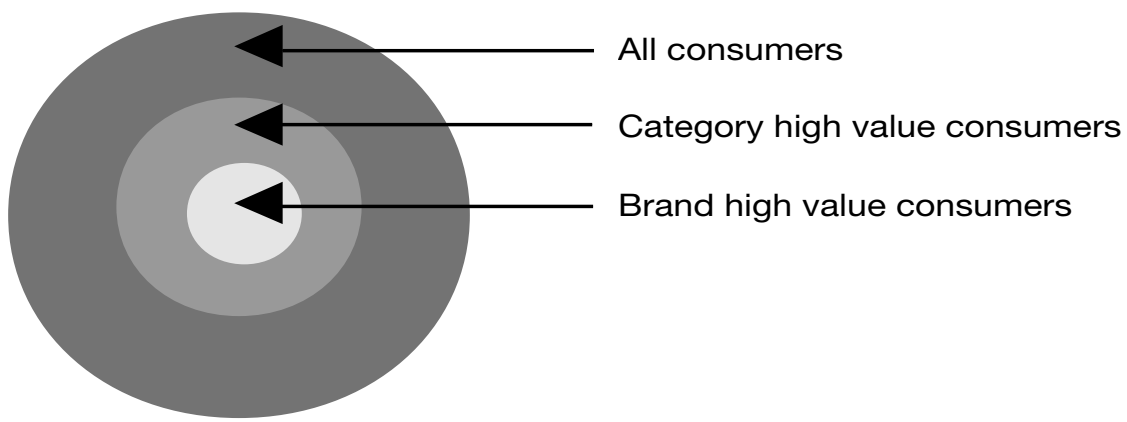

Figure 4: Consumer value categories

high category spenders have the same brand conversion propensity. From the standpoint of a given brand, a consumer's value should be considered high only if that consumer's category spending levels and brand conversion likelihood are both high. For example, in a category where $\$ 500$ in annual spending defines a high value consumer, a $\$ 1,000 /$ year consumer with a 60 per cent probability to convert to a given brand is a high value prospect to that brand, with the opposite being true of another $\$ 1,000 /$ year consumer with only 10 per cent brand conversion likelihood. It follows that, from the brand's point of view, a $\$ 2,000 /$ year consumer with only 10 per cent conversion likelihood is worth less than a $\$ 1,000 /$ year consumer with a 50 per cent conversion probability.

From a targeting point of view, new customer acquisition can be focused on all consumers, a subset of all consumers comprised of only 'high category value' consumers, or even a more homogenous subset of 'high brand value' consumers, as depicted in Figure 4.

Intuitively, the most specific target seems most appealing, which is not without a reason: those 'pre-qualified' consumers convert at a higher rate, are more profitable and can be more easily retained. Refocusing the brand's acquisition on brand high value consumers requires a couple of steps. First, an appropriate analytic frame of reference and decision metrics need to be identified. Start out by defining consumer value at the brand, not the category, level. It is unlikely that every heavy category spender will be recruited, so one should focus on identifying those heavy spenders that can be recruited. As a rule of thumb, the bulk of the marketing dollars should be invested in the middle of the consumer conversion continuum, with lower per capita investments allocated against both the least and the most likely to convert prospects. This is because investing heavily in the most likely to convert group represents unnecessary profit reduction, as those consumers are willing to buy the brand without much 'extra help', while spending heavily on the least likely to convert will do little to combat their loyalty to other brands or a lack of product-lifestage fit.

The second of the steps needed to refocus the brand on brand high value consumers entails the development of an explicit investment matrix. The starting point of this process is analytics intensive, entailing consumer segmentation and valuation to uncover conversion likelihood-based consumer segments. Next, each of the conversion segments needs to be profiled to support differential treatment strategies, which in turn involves varying investment levels and promotional tactics across segments. Of particular importance is the 

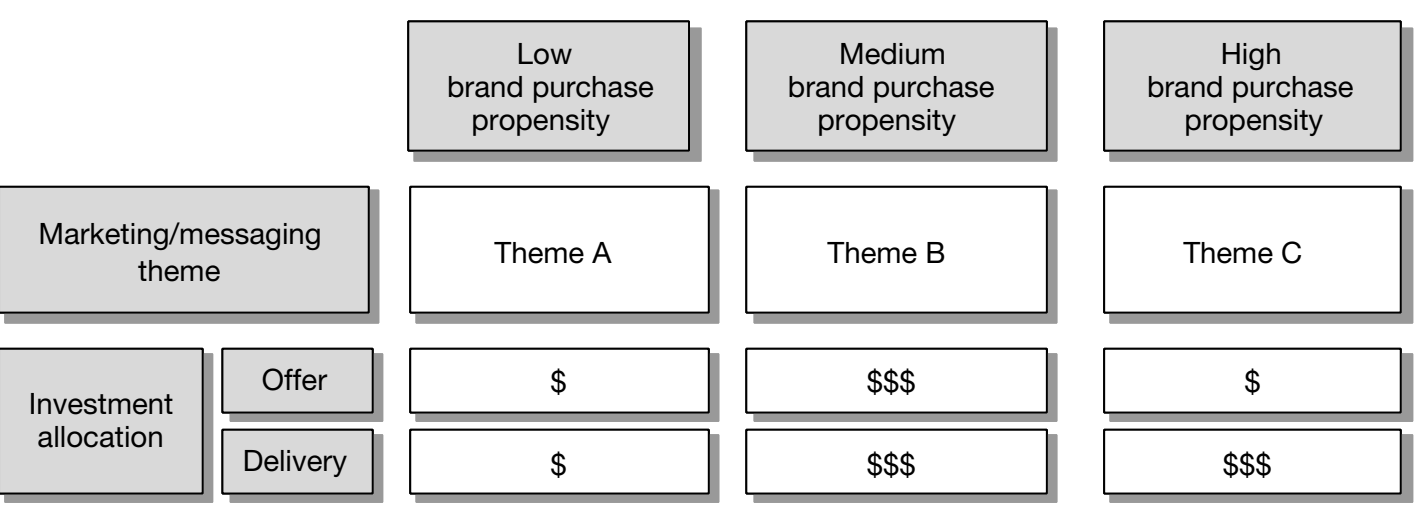

Figure 5: Sample investment matrix

segment-by-segment spending allocation. In general, the spending should be allocated keeping anticipated returns in mind, but proportional allotments should be avoided. In other words, the attractiveness of the high value segment should not be translated into over-investment into that segment. As a matter of fact, as previously suggested, the mid-range conversion likelihood segment is the most appropriate overinvestment target, as those consumers will likely show the highest incremental return on the additional spending. A general rule of thumb to help with setting the optimum selection threshold level is to pick a level where the relationship between total acquisition costs and the total consumer value is maximised. A sample investment matrix is shown in Figure 5 which depicts a common situation: a continuum of brand purchase propensity reduced to three action-suggestive categories placed in the context of two key sets of decisions promotional spending allocation across the three consumer value groupings and thematic messaging considerations.

Within the investment allocation dimension, it is important to differentiate between two separate considerations: offer-specific promotional spend allocation versus delivery-specific allocation. Offer spending refers to the cost of any purchase incentives, while delivery expenditures capture the cost of promotional materials, both on a per piece basis as well as in total (ie the number of contacts $X$ per piece cost). As shown in Figure 5, this distinction is important particularly for high brand purchase propensity prospects who do not require significant purchase incentives to convert, but whose interest in the brand should be reinforced with high quality materials and more frequent contacts.

\section{Summary of customer type-centric acquisition benefits}

The various advantages of the customer type-centric acquisition framework were described in the previous discussion. Figure 6 offers a concise summary, showing that decreasing per responder costs and increasing response rates are the most tangible benefits of the proposed acquisition framework. The per responder costs decline as a result of the lower number of targeted consumers once the 'low category value' and then the 'low brand value' consumers are eliminated from the target acquisition set. At the same time, the response rates climb, as more consumers react favourably to the offer on a percentage basis.

The following simple illustration serves to quantify potential savings. Consider 


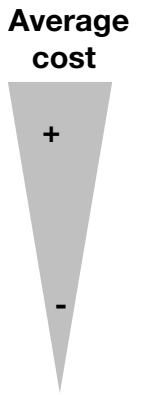

Acquisition approach
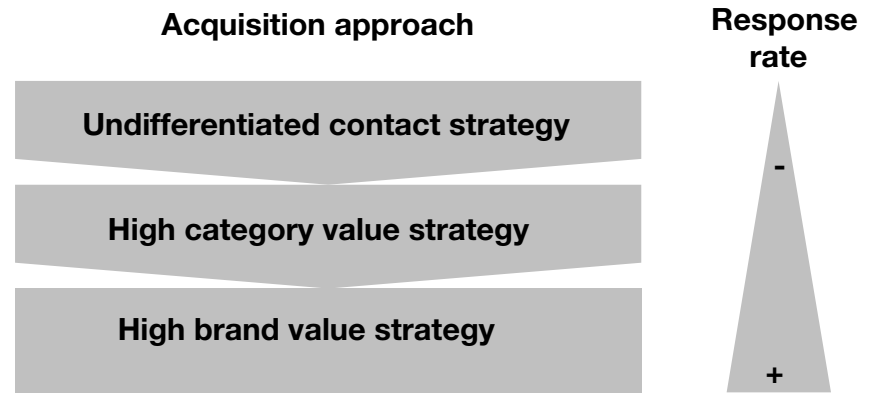

Figure 6: Acquisition type cost-benefit trade-offs

that for every 1,000 high category value consumers targeted, Brand A succeeds at converting 20 of them, all at the cost of $\$ 5$ per targeted consumer. This translates into a 2 per cent conversion rate and a total cost of $\$ 5,000$, or $\$ 250$ per converted consumer.

Brand $\mathrm{B}$, on the other hand, is frustrated by high costs and relatively low returns on its acquisition efforts and so decides to target only those high value consumers who exhibit the highest conversion likelihood. Rather than targeting all of the 1,000 high category spenders, Brand $\mathrm{B}$ decides to focus only on consumers whose conversion likelihood is greater than 50 per cent, which leads to a drop of 300 consumers from the original 1,000 (a very conservative estimate). Since the eliminated consumers were only those who were least likely to react positively, Brand $\mathrm{B}$ also converts 20 consumers, the same as Brand A, but there is a significant difference in the acquisition costs. Brand B's expenditures are considerably lower in terms of total costs $(\$ 3,500$ for Brand $B$ versus $\$ 5,000$ for Brand A); per acquired customer costs (\$175 for Brand B versus $\$ 250$ for Brand $\mathrm{A})$; and the conversion rate (2.9 per cent for Brand B versus 2 per cent for Brand A). But that's not all. A less (immediately) visible and more difficult to quantify benefit of the consumer-centric acquisition approach is a higher long term customer retention rate. As this might seem a bit less intuitive, a brief explanation might help.

As implied throughout this discussion, an initial product trial may be brought about by price incentives or a perceived fit between prospect needs and product benefits. It follows that the former will attract mostly price sensitive brand switchers while the latter will net a higher proportion of loyal repurchasers. Considering their deal-seeking orientation, switchers are easier to entice into price discounted product trials, but in the absence of continued price incentives they are also very likely to attrite. Overall, they have low per transaction profitability and dismal future value. Open offer-based acquisition programmes attract a higher proportion of these buyers than the customer type-centric approach. Adding to that the inherently more attractive profit and retention characteristics of prospects targeted by the latter, a clear picture emerges of a decisively better method. According to least one well-known source a 5 per cent increase in customer retention may lead to as much as a 75 per cent increase in long term profits. ${ }^{1}$

\section{CONCLUSION}

The basic premise of this paper is that the productivity of new customer acquisition can be considerably improved 
by migrating from a generic offer-centric approach to a customer type-centric approach. Poor response and repurchase rates can be lifted, soaring acquisition costs contained and transactional value improved, all leading to the heightening of longer term customer base profitability. The means to that end, as outlined in this paper, call for a fundamental redefinition of acquisition, away from a generic offer-based mass marketing oriented philosophy and towards a targeted, consumer insight driven model. Simply put, this means consumer information driven re-allocation and re-deployment of financial, creative and human acquisition resources to over-invest in high potential scenarios and under-invest in low potential scenarios. A number of well-known organisations in a variety of industries — including automotive, financial services and consumer products - are already enjoying two- to four-fold marketing productivity gains as a direct result of such transformations.

\section{References}

1 Reichheld, F. F. (1996) 'The Loyalty Effect: The Hidden Force Behind Growth, Profits, and Lasting Value', Harvard Business School Press, Cambridge, MA. 